\title{
Ad-dimers on Strained Carbon Nanotubes: A New Route for Quantum Dot Formation?
}

\author{
Daniel Orlikowski, Marco Buongiorno Nardelli, Jerry Bernholc, and Christopher Roland \\ Department of Physics, North Carolina State University, Raleigh, North Carolina, 27695
}

(Received 12 January 1999)

\begin{abstract}
The presence of ad-dimers on carbon nanotubes under tension leads to the formation of defects that wrap themselves around the circumference of the nanotube. These defects are actually short segments of a tube with a changed helicity, which suggests that the combination of ad-dimers plus strain can be used to form nanotube-based quantum dots. Our classical molecular dynamics and tight binding simulations show that the formation of such structures is particularly clean and promising for the $(n, 0)$ zigzag tubes, where the presence of ad-dimers induces plastic transformations on what are otherwise brittle tubes.

PACS numbers: 71.10.Hf, 71.20.Tx, 73.20.Dx
\end{abstract}

Among the many remarkable properties of single-walled carbon nanotubes, it is their mechanical and electronic properties that stand out [1]. The excellent resistance of carbon nanotubes to bending [2-7] should lead to future applications of the tubes as a high-strength, lightweight material. Turning to their electronic properties, we find that, at ambient temperatures, single-walled carbon nanotubes may be either metallic or semiconducting, depending upon their helicity [8-12]. It has been shown [13] that tubes with different helicities may be joined together with one or more pentagon-heptagon (5-7) defects to form different electronic heterojunctions, thereby opening up the intriguing possibility of forming all-carbon based microelectronic devices $[14,15]$. Some of these remarkable theoretical predictions have recently been confirmed by scanning tunneling microscopy (STM) experiments [16].

However, if the dream of producing an all-carbon-based microelectronics is ever to be realized, then different methods will be needed to produce a variety of devices. Here, we present the results of a large-scale study of the mechanical transformations of strained nanotubes in the presence of ad-dimers and show that this combination may well turn out to be a natural route for the formation of all-carbon nanotube-based quantum dots. Ad-dimers are likely to be present in small amounts on as-grown carbon nanotubes, or they may be deposited there with a STM tip or other methods. The formation of quantum dots with ad-dimers is particularly favorable for the $(n, 0)$ zigzag tubes. Specifically, we show that ad-dimers induce plastic behavior on tubes that are otherwise brittle.

Before discussing the simulations, we briefly review the behavior of pristine, strained carbon nanotubes. Beyond a critical value of the tension, estimated to be about $5 \%$, the system spontaneously releases its excess strain through a $90^{\circ}$ rotation of a $\mathrm{C}-\mathrm{C}$ bond around its center, the socalled Stone-Wales transformation [17]. This leads to the formation of a (5-7-7-5) defect, whose energetics has been investigated with both $a b$ initio and classical molecular dynamics simulations [18]. This defect furthermore acts as a nucleation center for the formation of dislocations and other nonlinear transformations [19,20]. Depending upon the external conditions and the helicity of the nanotubes, either brittle (the formation of cracks and their subsequent extension) or ductile (separation of dislocation cores through glide) behaviors are to be expected. In particular, brittle behavior is predicted for the large-diameter $(n, 0)$ zigzag tubes, and ductile behavior is predicted for the $(n, n)$ armchair tubes [20].

In our simulations, carbon atoms were modeled with a three-body Tersoff potential with a parametrization due to Brenner [21]. Recent studies of strained carbon nanotubes have demonstrated that there is good agreement between the results obtained with this potential and quantum simulations $[18,20]$. Some of our most critical results were confirmed with tight binding simulations.

Single adatoms adsorb to form handles between pairs of nearest-neighbor carbon atoms [22]. The adsorption energy for the adatoms varies weakly as a function of the nanotube diameter, ranging from $5.5 \mathrm{eV}$, on a $(5,5)$ tube with a $0.78 \mathrm{~nm}$ diam, to $4.9 \mathrm{eV}$ for a graphene sheet. The most important effect of adatoms on strained nanotubes is to reduce the activation energy for the StoneWales transformation. These activation energies are all uniformly reduced by $1.0-1.2 \mathrm{eV}$ for all strains [23]. This is due to an increased flexibility for the rotation of bonds that are next to the adatom handle, and implies that the presence of adatoms on strained nanotubes significantly enhances the rate of (5-7-7-5) defect formation.

We now turn to the action of carbon dimers. Figure 1 shows a typical dynamical evolution of a 3-nm-long $(480$ atoms $)(10,10)$ armchair tube with a carbon addimer at $2500 \mathrm{~K}$ and under a $3 \%$ strain. This ad-dimer initially sits on the surface of the nanotube. Within $4 \mathrm{ps}$, it is incorporated into the nanotube forming a novel defect consisting of back-to-back pentagons plus two heptagons, i.e., a (7-5-5-7) defect (Fig. 1a). This defect then undergoes further substantial evolution. After $356 \mathrm{ps}$, the bond emanating from the vertex of one of the pentagons and pointing away from the defect rotates to form a defect structure consisting of a single, rotated 
a)

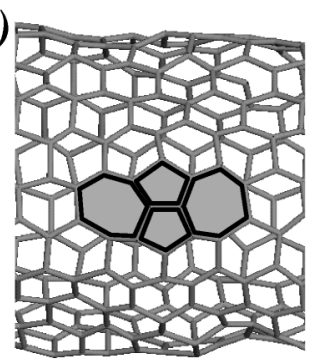

b)

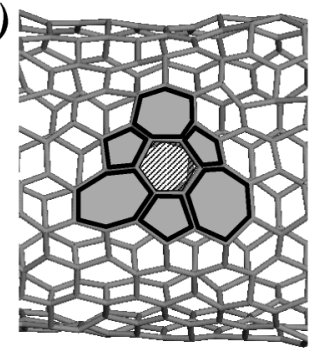

c)
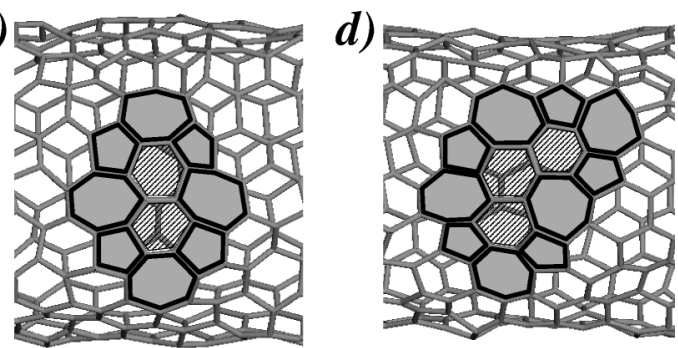

FIG. 1. Sample configurations showing the time evolution of a carbon ad-dimer on a $(10,10)$ tube under $3 \%$ strain: (a) (7-55-7) defect forms after $4 \mathrm{ps,} \mathrm{(b)} \mathrm{bond} \mathrm{rotation} \mathrm{leads} \mathrm{to} \mathrm{defect}$ with one rotated hexagon at $346 \mathrm{ps}$, (c) two hexagons at $421 \mathrm{ps,}$ and (d) three hexagons at $2.35 \mathrm{~ns}$.

hexagon that is separated from the rest of the nanotube through a "layer" of (5-7) pairs (Fig. 1b). Moreover, the creation of rotated hexagons continues: after $421 \mathrm{ps,} \mathrm{a}$ defect with two hexagons forms (Fig. 1c), while a third hexagon is incorporated after $2.35 \mathrm{~ns}$ (Fig. 1d). If this process of adding hexagons were to continue, the defect structure would eventually wrap itself completely around the circumference of the tube, forming a short segment of a nanotube with a different helicity.

The formation energies of these defects for the $(17,0)$ zigzag and $(10,10)$ armchair tubes are shown in Fig. 2. The formation energies are quoted for the most favored structures only. For example, there are at least two different ways in which the defect structure can wind itself around the nanotube, reflecting the hexagonal symmetry of the lattice. The rotation of a $\mathrm{C}-\mathrm{C}$ bond can be such that the newly formed hexagons wind themselves as directly as possible around the circumference of the tube or at $120^{\circ}$ to it. The formation energies reflect a preference for the former. This is because the rotation of a C-C bond is most efficient in relieving the strain if the bond is oriented perpendicular to the tension. Defects with large helical pitches necessarily involve the rotation of bonds that are at an angle with respect to the tube axis, and thus are less favorable. These trends are also reflected in the activation energies for the different bond rotations. For instance, for the $(10,10)$ tube with either a (7-5-5-7) defect or with a defect containing a single hexagon, at least one of the bonds emanating from the pentagons is directed perpendicular to the tension. Associated with the rotation of this bond are the activation energies of 3.8 (4.2 when

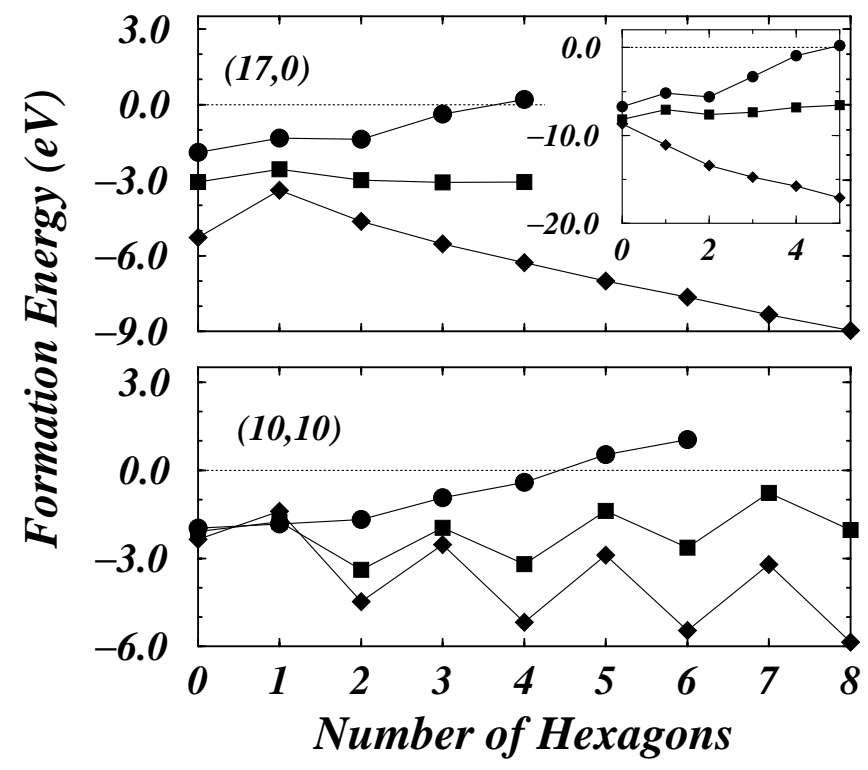

FIG. 2. Formation energies (measured with respect to two separated adatoms on the strained tubes) for the $(17,0)$ zigzag and $(10,10)$ armchair tubes versus the number of hexagons incorporated in the defect. The symbols mark different levels of strain for the tubes: circles, 0\%; squares, 5\%; diamonds, $10 \%$. The inset shows corresponding tight binding results for the $(17,0)$ tube.

single hexagon is present), 3.2, and $2.3 \mathrm{eV}$ for $0 \%, 5 \%$, and $10 \%$ strains, respectively. When this bond is at an angle, as is the case when two hexagons are present, the activation increases to $4.2-4.4 \mathrm{eV}$ for all of the strains. Activation energies for the $(17,0)$ tube are $4.1,3.7$, and $2.8 \mathrm{eV}$ for the same strains, respectively, and vary only weakly as the number of hexagons in the defect structure is increased [23].

From the graph, it is clear that the formation of the defect structures is intimately related to the strain. In the absence of strain, the formation energy of the (7-5-5-7) defect is lowest for both the $(10,10)$ and $(17,0)$ tubes, indicating that structures with rotated hexagons are not to be expected. We now consider the behavior of the strained $(10,10)$ tube. Under a $5 \%$ strain, the defect with two rotated hexagons has the lowest energy, indicating that structures containing more hexagons represent transient, metastable configurations. Under a $10 \%$ strain, the formation energy decreases as the number of hexagons in the defect increases, showing that larger strains lead to the wrapping of the defect around the tube. Note also that the formation energy of the $(10,10)$ tube oscillates with the number of hexagons it contains. This is simply a reflection of the geometry of the armchair tubes. For a defect containing an even number of hexagons, the bonds that need to be rotated in order to incorporate the next hexagon are all at an angle with respect to the ones already present (Figs. 1c and 1d), so that the hexagon must necessarily be formed next to rather than directly above the existing 
hexagons. This behavior is to be contrasted with that of the strained $(17,0)$ zigzag tube. Here, there are no oscillations in the variation of energy, indicating that hexagons are all added to the structure in a similar manner, as is seen by comparing Figs. 1 and 3. Under 5\% strain, the defect with three hexagons has the lowest energy. However, the energy differences between adjacent configurations are quite small, so that energetically the structures are nearly degenerate. Structures with a larger number of hexagons may therefore readily be formed at finite temperatures. Under the larger $10 \%$ strain, the formation energy now decreases continuously so that the wrapping of the defect around the circumference of the nanotube is favored. Note that qualitatively similar results are obtained with a more accurate tight binding model [24], as shown in the inset of Fig. 2 [25].

The winding of the defect around the nanotube suggests that the combination of ad-dimers plus strain in the $5 \%-10 \%$ range may be a natural way to produce different electronic heterojunctions, thereby forming different quantum dots. Indeed, the formation of such interfaces based on strain alone has previously been suggested [1820]. Such strains are achievable via a combination of bending and manipulation. Recently, such techniques have been applied to nanotubes in the context of an investigation of the electrical response of strained carbon nanotubes [26]. We note that nanotube-based quantum dots have also been obtained using a nanotube/ $\mathrm{SiC}$ nanorod interface [27]. However, in contrast to this and other methods based on chemical functionalization, the approach proposed here has the advantage of producing pure all-carbon-based heterojunctions with a controlled geometry.

To test this idea, we constructed various ad-dimerbased defect structures at different strains and annealed them at temperatures in the $2500-3000 \mathrm{~K}$ range [28]. The
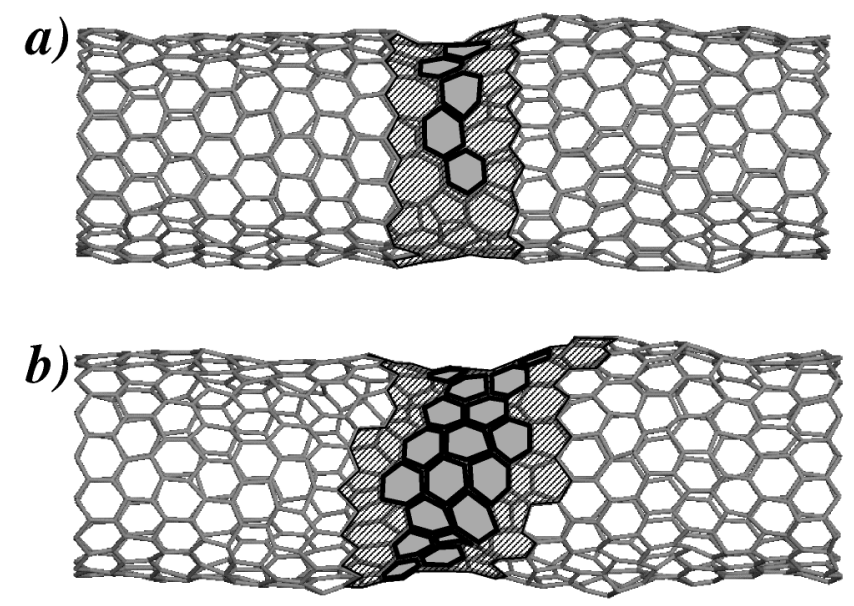

FIG. 3. Time evolution of the $(17,0)$ tube with an ad-dimer under $7.5 \%$ strain at $3000 \mathrm{~K}$, illustrating the spontaneous winding of the defect around the tube: (a) the initial configuration consisting of a single turn, and (b) the final configuration corresponding to about three turns after $1 \mathrm{~ns}$. following results were obtained. Structures on the $(10,10)$ armchair tubes were not observed to be stable. Competing bond rotations (e.g., Stone-Wales rotations away from the defect, or bonds on the heptagons) lead to the degradation of the structure within a few nanoseconds. It therefore seems quite unlikely that good quantum dots can be made with the help of ad-dimers from the $(10,10)$ and/ or other armchair tubes. Much more promising results are obtained for the $(17,0)$ zigzag tube. Figure 3 shows sample configurations of such a tube consisting of 682 atoms, annealed at $3000 \mathrm{~K}$ and $7.5 \%$ strain. As is evident, there is no sign of any competing ductile behavior that would lead to the degradation of the structure. Rather, hexagons are added in a uniform fashion around the circumference of the tube, ultimately leading to the formation of two to three different windings of an $(8,8)$ tube over the period of a nanosecond. This suggests that with the addition of ad-dimers to strained zigzag tubes one can selectively induce ductile behavior on tubes that are otherwise brittle, and thus form clean interfaces between tubes of different helicities. Therefore, provided one can add enough ad-dimers, the map of brittle versus ductile behavior as a function of helicity [20] is altered such that all tubes display ductile behavior.

To characterize the electronic properties of the induced $(17,0) /(8,8) /(17,0)$ structure we have calculated the local density of states (LDOS) using a recursion method [29] within a tight binding description of the carbon $\pi$ bonds. Only nearest-neighbor interactions were considered, with the two-center hopping integral $V_{p p \pi}=-2.66 \mathrm{eV}$ [13]. Figure $4 \mathrm{a}(4 \mathrm{~d})$ shows the LDOS of a pure $(17,0)$ semiconducting $[(8,8)$ metal] tube, while Figs. $4 \mathrm{~b}$ and $4 \mathrm{c}$ show results for the LDOS for both the initial and final structures shown in Fig. 3. These were obtained by averaging contributions from all atoms that constitute the defect, including those comprising the (5-7) pairs that separate the small $(8,8)$ segment from the $(17,0)$ tubes. For this semiconductor/metal heterojunction, there is a considerable enhancement of the LDOS near the Fermi level, reflecting the large contribution from the interface states, which clearly dominate Fig. 4b. The contribution of these states decreases considerably in magnitude as the length of the $(8,8)$ segment increases (Fig. 4c). Ultimately, the emergence of a quantum dot structure with localized interface states is to be expected [15].

In summary, we have investigated the mechanisms of strain relief of carbon nanotubes with either adatoms and/ or ad-dimers under tension. The presence of adatoms lowers the activation energies for the Stone-Wales rotation significantly, so that the rate of the (5-7-7-5) defect formation is enhanced. Ad-dimers induce the formation of a new set of defects, consisting of rotated hexagons separated by (57) pairs from the rest of the tube. As these defects wind themselves around the tubes, the combination of ad-dimers plus strain provides a natural route for the formation of different quantum dots. The best results are achievable for 


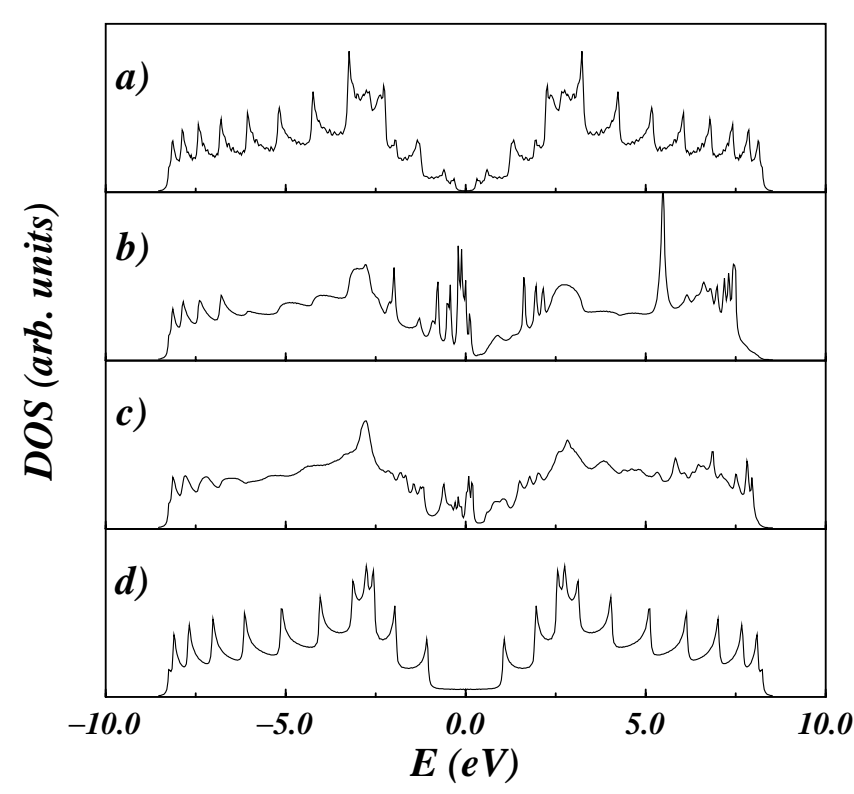

FIG. 4. LDOS for the strained $(17,0)$ tube with an ad-dimer: (a) ideal $(17,0)$, (b) for configuration shown in Fig. 3a, (c) for configuration shown in $3 \mathrm{~b}$, and $(\mathrm{d})$ ideal $(8,8)$ tube. Note that $0 \mathrm{eV}$ marks the position of the Fermi level.

$(n, 0)$ zigzag tubes with $n \geq 14$ (or tubes with chiralities close to zigzag). For these tubes, one can take advantage of their normally brittle behavior [20] and induce plastic transformations with ad-dimers in a controlled and usable fashion.

This work was supported in part by Grants No. ONR N00014-98-1-0597 and NASA NAG8-1479. We also thank the North Carolina Supercomputing Center (NCSC) for computer support.

[1] R. F. Service, Science 281, 940 (1998).

[2] S. Iijima, C. Brabec, A. Maiti, and J. Bernholc, J. Chem. Phys. 104, 2089 (1992).

[3] B. I. Yakobson, C. J. Brabec, and J. Bernholc, Phys. Rev. Lett. 76, 2511 (1996).

[4] M. R. Falvo, G. J. Clary, R. M. Taylor II, V. Chi, F. P. Brooks, Jr., S. Washburn, and R. Superfine, Nature (London) 389, 582 (1997).

[5] E. Hernandez, C. Goze, P. Bernier, and A. Rubio, Phys. Rev. Lett. 80, 4502 (1998).

[6] J. Despres, E. Daguerre, and K. Lafdi, Carbon 33, 87 (1995).

[7] N. Chopra, L. Benedict, V. Crespi, M. L. Cohen, S. G. Louie, and A. Zettl, Nature (London) 377, 135 (1995); R. Ruoff and D. Lorentz, Bull. Am. Phys. Soc. 40, 173 (1986).

[8] J. W. Mintmire, B. I. Dunlap, and C. T. White, Phys. Rev. Lett. 68, 631 (1992).

[9] N. Hamada, S. I. Sawada, and A. Oshiyama, Phys. Rev. Lett. 68, 1579 (1992).
[10] R. Saito, M. Fujita, G. Dresselhaus, and M. S. Dresselhaus, Appl. Phys. Lett. 60, 2204 (1992); Phys. Rev. B 46, 1804 (1992).

[11] X. Blase, L. X. Benedict, E. L. Shirley, and S. G. Louie, Phys. Rev. Lett. 72, 1878 (1994).

[12] For an analysis of some many-body effects, see, for example, C. L. Kane and E. J. Mele, Phys. Rev. Lett. 78, 1932 (1997); Y. A. Krotov, D.-H. Lee, and S. G. Louie, ibid. 78,4245 (1997); L. Balents and M.P. A. Fischer, Phys. Rev. B 55, R11973 (1997).

[13] L. Chico, V.H. Crespi, L.X. Benedict, S. G. Louie, and M. L. Cohen, Phys. Rev. Lett. 76, 971 (1996); L. Chico, L. X. Benedict, S. G. Louie, and M. L. Cohen, Phys. Rev. B 54, 2600 (1996).

[14] V.H. Crespi, M. L. Cohen, and A. Rubio, Phys. Rev. Lett. 79, 2093 (1997); J. C. Charlier, T.W. Ebessen, and Ph. Lambin, Phys. Rev. B 53, 11108 (1996).

[15] L. Chico, M.P. Sancho, and M. C. Munoz, Phys. Rev. Lett. 81, 1278 (1998).

[16] J.W. G. Wildoeer, L. C. Venema, A. G. Rinzler, R.E. Smalley, and C. Dekker, Nature (London) 391, 59 (1998); J. W. Odom, J.-L. Huang, P. Kim, and C. M. Lieber, ibid. 391, 62 (1998).

[17] A. J. Stone and D. J. Wales, Chem. Phys. Lett. 128, 501 (1986).

[18] M. Buongiorno Nardelli, B. I. Yakobson, and J. Bernholc, Phys. Rev. B 57, R4277 (1998).

[19] B. I. Yakobson, Appl. Phys. Lett. 72, 918 (1998).

[20] M. Buongiorno Nardelli, B. I. Yakobson, and J. Bernholc, Phys. Rev. Lett. 81, 4656 (1998).

[21] J. Tersoff, Phys. Rev. Lett. 61, 2879 (1988); Phys. Rev. B 37, 6991 (1988); D. Brenner, Phys. Rev. B 42, 9458 (1990).

[22] A. Maiti, C. J. Brabec, and J. Bernholc, Phys. Rev. B 55, 6097 (1997).

[23] D. Orlikowski, M. Buongiorno Nardelli, J. Bernholc, and C. Roland (unpublished).

[24] C. H. Xu, C.Z. Wang, C. T. Chan, and K. M. Ho, J. Phys. Condens. Matter 4, 6047 (1992).

[25] The difference in the energy scales as obtained from the classical and tight binding models is due to the range over which the bond lengths are perturbed by the defects. An examination of the $\mathrm{C}-\mathrm{C}$ bond lengths shows that a much greater number of atoms are perturbed in the tight binding model than in the classical models, accounting for the energy differences.

[26] S. Paulson, M. R. Falvo, N. Snider, A. Helser, T. Hudson, A. Seeger, R. M. Taylor II, R. Superfine, and S. Washburn, http://xxx.lanl.gov/abs/cond-mat/ 9905304/preprint (1999).

[27] J. Hu, P. Yang, and C. M. Lieber, Nature (London) 399, 48 (1999); Y. Zhang, T. Ichihashi, E. Landree, F. Nikey, and S. Iijima, Science 285, 1719 (1999).

[28] The large temperatures are dictated by the relatively short time scales achievable in a molecular dynamics simulation. However, we fully expect similar transformations to occur at the lower, experimentally achievable temperatures, albeit on a longer time scale.

[29] R. Haydock, V. Heine, and M. J. Kelly, J. Phys. C 8, 2591 (1975). 